\title{
A conceptual framework for mapping uncertainty in integrated assessment
}

\author{
$\underline{\text { T. Oxley }}^{a}$ and H. ApSimon ${ }^{\text {a }}$ \\ ${ }^{a}$ Centre for Environmental Policy, Faculty of Natural Sciences, \\ Imperial College London, South Kensington, London, SW7 2AX, United Kingdom \\ Email:t.oxley@imperial.ac.uk
}

\begin{abstract}
At a time when Integrated Assessment Modelling is increasingly providing the scientific basis for policy development in relation to air quality and climate change, scientists and modellers are facing a dilemma: How can we effectively address uncertainty? Whereas policy makers demand quantifications of uncertainty from these state-of-the-art models, the increasingly complex and inter-dependent scientific domains and spheres of human activity captured by the models means that scientists can rarely provide better than qualitative representations of uncertainty. Using the context of the UK Integrated Assessment Model we present a conceptual framework within which uncertainties in different components of integrated assessment models can be classified. We show how an hierarchy of uncertainties can be identified which will assist policy makers in understanding where an uncertainty arises and to what extent it may impact upon policy development. Policy makers must still make the decisions. This generic conceptual framework will help scientists and modellers to provide policy makers with an understanding of uncertainties involved whilst highlighting that models are only heuristic tools designed to help make the decision and understand the potential impacts of that decision in an inherently uncertain world.
\end{abstract}

Keywords: Integrated Assessment; Uncertainty; Conceptual Framework; UKIAM 


\section{INTRODUCTION}

At a time when Integrated Assessment Modelling (IAM) is increasingly providing the scientific basis for policy development in relation to air quality and climate change, scientists and modellers are facing a dilemma: How can we effectively address uncertainty? Whereas policy makers demand quantifications of uncertainty from these state-of-the-art models, the increasingly complex and inter-dependent scientific domains and spheres of human activity captured by the models means that scientists can rarely provide better than qualitative representations of uncertainty. To emphasise this problem the EC4MACS uncertainty review concluded that "the greatest uncertainty is in quantification of the uncertainties themselves." [EC4MACS, 2010]. Moreover, uncertainty tends to be addressed in a very fragmented way; either quantitatively or qualitatively; usually only in parts of the models (eg. emissions inventories; costs/benefits etc.); often ambiguously and confused with model sensitivities or scenario analyses; and probably inadequately.

Current techniques for addressing uncertainty in the context of policy development include HAZard and OPerability studies (HAZOP), which can be used as a risk assessment technique to help identify areas of uncertainty, including assumptions and external factors are not directly modelled, for example in negotiation of the Gothenburg Protocol [ApSimon et al., 2002]. Other techniques use uncertainty matrices which can capture knowledge related uncertainty [Walker et al., 2003; Petersen, 2006], or Artificial Neural Networks which analyse a range of parameter values for multiple influences [Carnevale et al., 2009]. Cullen \& Frey (1999) provide an extensive handbook of probabilistic techniques for exposure assessment.

Using the context of the UK Integrated Assessment Model (UKIAM) [Oxley et al., 2003a; Oxley \& ApSimon, 2007] - developed under the auspices of the UN/ECE Convention on Long-Range Transboundary Air Pollution (CLRTAP) - we present a conceptual framework within which uncertainties in different components of integrated assessment models can be classified (empirical, mathematical, virtual, perceptual etc.).

\section{CURRENT LITERATURE}

Since uncertainty exists at all levels from the empirical through to value-driven responses to policy it is not possible to present an exhaustive review of the literature in this paper. However, others have published extensive literature reviews in more focussed aspects of integrated assessment such as Critical Loads [Skeffington, 2006] or road traffic forecasts [Jong et al., 2007].

The diversity of different data and simulation based components which constitute an integrated assessment model such as the UKIAM, GAINS [Amann et al., 2011] or other similar models (for example, see http://niam.scarp.se/), has given rise to a plethora of literature covering specific aspects of integrated assessment. These uncertainty assessments include parameter uncertainty in atmospheric dispersion models [Alcamo \& Bartnicki, 1987; Tost et al., 2010], atmospheric chemistry and nitrogen deposition [Derwent, 1987; Sutton et al., 2008], Critical Loads and natural ecosystems [Reinds \& de Vries, 2010; Skeffington, 2006; Wolniewicz \& Aherne, 2010; Zak et al., 1997; http://cldm.defra.gov.uk/Uncertainties.htm] and uncertainties in national emissions inventories [Passant, 2003; Bush et al., 2010]. In relation to road transport alone there are many assessments, for example in relation to transport emissions [Kioutsioukis et al., 2004; Kouridis et al., 2010], non-exhaust emissions [Stocker \& Carruthers, 2007] and traffic forecasts [Jong et al., 2007; Zhao \& Kockelman, 2001], whereas de Haan \& Keller (2004) present an approach to modelling fuel consumption which attempts to avoid uncertainties resulting from individual driving behaviours. In relation to integrated assessment models Schoepp et al. (2005) address the uncertainty of emissions estimates in the RAINS model, and Oxley et al. (2011) highlight uncertainties which emerge from the integration of models of spatial resolutions.

However, few of these uncertainty assessments (with exceptions such as Mathijssen et al., (2007)) adequately address the potential significance of uncertainties in the context of trans-disciplinary policy development and the often far greater uncertainties in scenario development and the interpretation of effects by policy makers. For this reason the framework described below shows how an hierarchy of uncertainties can be identified which will assist policy makers in understanding where an uncertainty arises (monitoring data, process representation, scenario definition etc.) and to what extent it may impact upon policy development.

\section{TOWARDS A CONCEPTUAL FRAMEWORK}

In preference to trying to always quantify uncertainties there should be an attempt to understand the implications of uncertainties from a given perspective (in this case policy making supported by IAM). It is of course significant where different uncertainties occur. Uncertainties in deposition rates for different vegetation may be quantifiable and of high significance to atmospheric dispersion modellers (eg. Sutton et al., (2008)) but these may have little impact upon scenario analyses where there may be greater uncertainties in projected emissions, scenario definitions and abatement costs. In the former there is interest in increasing the precision of dispersion models whereas in the latter the interest will be more in the reality of deposition patterns in relation to the scenarios and potential impacts. A conceptual framework within which different types of uncertainties can 
be located and put in context in relation to the observer's perspective is therefore helpful in communicating an understanding of the implications of uncertainties to policy makers.

A conceptual framework developed in the context of hydrological resources and desertification in the Mediterranean [Oxley et al., 2003b] provides the basis for the conceptual framework for understanding uncertainty described here (Figure 1). Similar issues are relevant for effective communication of scientific meaning to policy makers. Whereas any component of IAM can be classified as empirical (data), theoretical (models), virtual (IAM's) or perceptual (scenarios), the same component can be identified as contributing to historical memory or projected futures, and/or providing utility in either decision support or communication. Finally, Hammond's (1978) six modes of thought capture all IAM components from the highly experimental (data), through analytical (modelling) to highly intuitive (policy scenarios). This framework has been used as the basis for classifying IAM components elsewhere [Oxley \& ApSimon, 2007]. The categories described by Figure 1 are not rigid but serve to distinguish characteristics of different components of IAM. For example, whereas empirical studies may be analytical, can describe historical conditions, are useful for communication, and provide raw data for models, they cannot directly address projected futures; this is achievable indirectly by the use of empirical data by models (theoretical) and decision support tools (virtual).

Within this framework the different areas of uncertainty in IAM can begin to be located, identifying which uncertainties are directly related to the overall model itself (UKIAM), component models (FRAME dispersion modelling), input data (emissions) etc. An example of such a distinction is provided by Amann et al. (2011) in relation to the GAINS model where source-receptor matrices from EMEP provide the basis for defining reduced-form approximations within GAINS. There is uncertainty in these approximations $\left(\mathrm{R}^{2} \approx 0.99\right)^{[1]}$ but only in relation to the EMEP calculations; these uncertainties are attributable to GAINS, but any uncertainties (eg. atmospheric chemistry) in the EMEP calculations themselves are not. This highlights the need to be explicit about both the perspective of the observer and how different uncertainties relate to that perspective.

\section{MAPPING THE UKIAM ONTO THE CONCENTUAL FRAMEWORK}

Some of the model components creating uncertainties captured by and/or attributable to the UKIAM (and the BRUTAL sub-model for road transport [Oxley et al., 2009]) are listed in Table 1, classifying them in relation to source, type, use etc. Thus we can identify attributes of each area of uncertainty in relation to the conceptual framework. Uncertainties may be definable as:

- $\quad$ Empirical (eg monitoring data, distribution of emissions)

- Theoretical (eg mathematical representations/models)

- Virtual (eg. Integrated Assessment Models, output metrics)

- $\quad$ Perceptual (eg. Scenario definitions, emissions projections, policy impacts etc.)

${ }^{[1]}$ The coefficient of determination $R^{2}$ is used in the context of statistical models whose main purpose is the prediction of future outcomes on the basis of other related information. It provides a measure of how well future outcomes are likely to be predicted by the model. 
In addition the uncertainties may relate to:

- Historical memory (eg. monitoring data, expert opinion, perceptions)

- Projected futures (eg. climate change, human activity, economics, models)

- Decision support mechanisms (modelling, data, IAM, etc.)

- Communication mechanisms (eg. IAM, policy fora, UNECE, media, monitoring)

\begin{tabular}{|c|c|c|c|c|c|c|c|c|c|c|c|}
\hline & 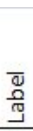 & Nature of Uncertainty & Source & Type & $\begin{array}{l}\text { Relationship to } \\
\text { UKIAM }\end{array}$ & 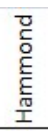 & $\stackrel{n}{\frac{n}{d}}$ & 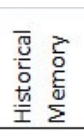 & 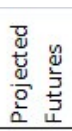 & 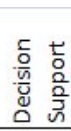 &  \\
\hline \multirow{14}{*}{ UKIAM } & A & Spatial distribution of emissions & NAEI & Empirical & Input data & $\mathrm{I}$ & $P$ & $\checkmark$ & & & $\checkmark$ \\
\hline & B & Projected emissions by source & NAEI & Theoretical & Input data & IIII & $\mathrm{R}$ & & $\checkmark$ & & $\checkmark$ \\
\hline & c & Source stack heights & NAEI & Empirical & Input data & 1 & $\mathrm{P}$ & $\checkmark$ & & & $\checkmark$ \\
\hline & D & Atmospheric Dispersion & FRAME/PPM & Theoretical & Input data (SRM's) & IIII & $\mathrm{R}$ & $\checkmark$ & & $\checkmark$ & \\
\hline & $\mathrm{E}$ & Atmospheric Chemistry & FRAME & Theoretical & External (FRAME) & "I & G & $\checkmark$ & & $\checkmark$ & \\
\hline & $\mathrm{F}$ & Deposition Rates & FRAME & Theoretical & External (FRAME) & "I & G & $\checkmark$ & & $\checkmark$ & \\
\hline & G & Spatial distribution of ecosystems & $\mathrm{CEH} / \mathrm{JNCC}$ & Empirical & Input data & 1 & $\mathrm{p}$ & $\checkmark$ & & $\checkmark$ & \\
\hline & $\mathrm{H}$ & Ecosystem Critical Loads & CEH & Experimental & Input data & $1 / 11$ & G & $\checkmark$ & & $\checkmark$ & \\
\hline & 1 & Imported Contributions & ASAM/EMEP & Virtual & Input data (ASAM) & IIII & $\mathrm{R}$ & & $\checkmark$ & $\checkmark$ & \\
\hline & $\mathrm{J}$ & Scenario definition & Defra/DECC & Perceptual/Virtual & Scenario Setup & $\mathrm{v}$ & $\mathrm{R}$ & & $\checkmark$ & & $\checkmark$ \\
\hline & $\mathrm{k}$ & Critical Loads Exeedance & UKIAM & Virtual & Output metric & IV & $\mathrm{R}$ & & $\checkmark$ & & $\checkmark$ \\
\hline & L & Total Deposition & UKIAM & Virtual & Output metric & IV & $\mathrm{R}$ & & $\checkmark$ & & $\checkmark$ \\
\hline & M & Aerosol Concentrations & UKIAM & Virtual & Output metric & IV & $\mathrm{R}$ & & $\checkmark$ & & $\checkmark$ \\
\hline & $\mathrm{N}$ & Background PM/Resuspension etc. & AEA/ASAM & Empirical & Input data & $1 / 11$ & $\mathrm{R}$ & $\checkmark$ & & $\checkmark$ & \\
\hline \multirow{10}{*}{ BRUTAL } & 0 & Average road speeds & Estimated & Theoretical & Input data & II & G & $\checkmark$ & & $\checkmark$ & \\
\hline & $\mathrm{P}$ & Projected traffic flows & DfT/LAEI & Perceptual & Scenario Setup & $\mathrm{v}$ & $\mathrm{R}$ & & $\checkmark$ & & $\checkmark$ \\
\hline & Q & Population distribution & CENSUS & Empirical & Input data & 1 & $\mathrm{P}$ & $\checkmark$ & & $\checkmark$ & \\
\hline & $\mathrm{R}$ & Street Canyon definitions & Estimated & Theoretical & Input data & "I & G & $\checkmark$ & & $\checkmark$ & \\
\hline & $\mathrm{s}$ & Speed-dependent emissions factors & NAEI & Empirical/Theoretical & Input data & II & $\mathrm{R}$ & $\checkmark$ & & $\checkmark$ & \\
\hline & $\mathrm{T}$ & $\mathrm{f}-\mathrm{NO}_{2}$ emissions & NAEI/AQEG & Empirical & Input data & II & $\mathrm{P}$ & $\checkmark$ & & $\checkmark$ & \\
\hline & u & $\mathrm{NO}_{x} / \mathrm{NO}_{2}$ Chemistry & AQEG & Theoretical & Calc'd by BRUTAL & III & G & $\checkmark$ & & $\checkmark$ & \\
\hline & $\mathrm{v}$ & Fleet/Technology Mix & AEA/Dft & Perceptual & Scenario Setup & $\mathrm{v}$ & $\mathrm{R}$ & & $\checkmark$ & & $\checkmark$ \\
\hline & w & Roadside Concentrations & BRUTAL & Virtual & Output metric & IV & $\mathrm{R}$ & & $\checkmark$ & & $\checkmark$ \\
\hline & $\mathrm{x}$ & Background Concentrations & BRUTAL & Virtual & Output metric & IV & $\mathrm{R}$ & & $\checkmark$ & & $\checkmark$ \\
\hline \multirow{2}{*}{ EXTERNAL } & $\mathrm{Y}$ & AQ Monitoring Data & AEA/LAEI & Empirical & Validation & 1 & $\mathrm{P}$ & $\checkmark$ & & & $\checkmark$ \\
\hline & $\mathrm{z}$ & Scenario Interpretation & Policy Makers & Perceptual & Effects & $\mathrm{VI}$ & $\mathrm{R}$ & $\checkmark$ & & & $\checkmark$ \\
\hline
\end{tabular}

Table 1 - Categorisation of selected components of the UKIAM displaying different areas of uncertainty, distinguishing the source, type (empirical, theoretical etc.), the mode of thought associated with the uncertainty [Hammond, 1978] ], the tendency of the component towards reality, generality or precision [Levins, 1966], the utility of the component in relation to decision support or communication, and temporal perspective of the information generated.

Finally, depending upon the observer, their 'mode of thought' may vary from highly analytical through experimental to highly intuitive, potentially greatly influencing the perceived significance of each of the other attributes, the balance between the need for precision of data, realistic results and generic applicability [Levins, 1966], and their requirement for quantifiable or qualifiable interpretations of uncertainty.

A further degree of complexity becomes evident when examining potential emissions abatement measures, where uncertainties may arise in the specification of activity rates, existing abatement technology, and applicability and effectiveness of additional measures.

Thus, an ability to conceptually map uncertainties will begin to identify which aspects of uncertainty in the overall 'system' are of significance to the observer. It also facilitates the definition of sensitivity scenarios which may quantify the extent to which uncertainties within the system may impact upon the overall results. For example running scenarios with calibrated or un-calibrated FRAME data (see Fournier et al. (2004)) would highlight the sensitivity of UKIAM to FRAME calculations and place uncertainties (both quantifiable and qualifiable) in FRAME in the context of potential impact upon UKIAM outputs.

Having listed selected areas of potential uncertainty these can now be 'located' on a conceptual map (Figure 1) with an additional level of classification which assumes that empirical components aim to maximise precision, modelling components aim to maximise generic applicability, and scenario/output components aim to maximise reality; an objective of integrated assessment models is to provide an appropriate balance between reality, precision and generality which best communicates the potential impacts of different scenarios to policy makers.

Using the labels defined in Table 1 these components displaying uncertainties are mapped onto the conceptual framework (Figure 2) highlighting the dependencies between components. Separate maps are presented for UKIAM and BRUTAL to show how multiple layers of uncertainty maps can be created to capture the many different tasks required to complete a fully integrated assessment of any given policy scenario. Using the 
FRAME model (component D in Table 1) as an example, empirical data underpins the definition of the spatial distribution (A) and release height (C) of emissions, a combination of empirical and experimental information determines the representation by FRAME of atmospheric chemistry (E) and deposition rates (F), all of which are combined to derive atmospheric dispersion (D) patterns; it is these dispersion/deposition patterns that are used by UKIAM to calculate total deposition (L), aerosol concentrations (M) and thus exceedance of critical loads (K). Additional conceptual maps could be produced to capture, for example:

- definition of abatement measures based upon activity levels, technology efficiency, applicability etc.;

- the FRAME model to more fully investigate uncertainties in atmospheric dispersion;

- the ASAM model to understand the effects of uncertainties at the European scale (which feed into Figure 2 as 'imported contributions'); or

- other areas where uncertainties are evident (eg. energy, transport or agricultural livestock projections which underlie emissions projections).

Thus it is possible to build up multiple layers of inter-related uncertainty maps which all ultimately feed into the interpretation of model outputs for given policy scenarios.


Figure 2 - Mapping of the selected components onto the conceptual framework, distinguishing between (a) the UKIAM and (b) the BRUTAL sub-model. An additional level of classification is highlighted to emphasise which components aim (non-exclusively) to maximise precision, which aim to maximise generality, and which are required to be appropriately realistic. The lettering of components reflects the labelling described in Table 1. Colours have been used to highlight how some different components may be linked.

\section{DISCUSSION}

The mapping described above is for explanatory purposes only, identifying selected areas of uncertainty within the scope of the UK Integrated Assessment Model. Clearly there are additional layers of uncertainty maps which could be included, focussing on the definition of ecosystem distribution $(\mathrm{G})$ or specification of Critical Loads $(\mathrm{H})$, the FRAME model (D, E, F) and the influence of spatial distribution (A) and height (C) of emissions [Vieno et al., 2010], traffic forecasting (P, V), abatement measures [Entec, 2009] or the ASAM model [Warren \& ApSimon, 1999; Oxley \& ApSimon, 2007]. The complexity of inter-relationships between each conceptual map and the potential 'depth' of uncertainties within the system relative to results interpreted by policy makers (Z) quickly becomes evident; little wonder it has been concluded that the "greatest uncertainty is in quantification of uncertainty itself"' [EC4MACS, 2010].

However, the conceptual framework presented here overcomes this problem by presenting relationships between areas of uncertainty in a visual form. Quantifications of uncertainties in specific areas are always of benefit (eg. Vieno et al., 2010). The effect of the spatial distribution and height of emission can be quantified in relation to source-receptor matrices calculated by the FRAME model but not in relation to the use by the UKIAM of these matrices. In the latter case the sensitivity of the UKIAM to different footprints, ceteris paribus ${ }^{[2]}$, can be assessed. This cannot equate to a quantification of uncertainty since the sensitivity of the UKIAM to different representations of Critical Loads $(\mathrm{H})$ or uncertainties in ecosystem distribution $(\mathrm{G})$ is likely to be different for the same degrees of uncertainty displayed within the different components.

[2] "all other things being equal or held constant" 
The sensitivity of UKIAM to FRAME source-receptor matrices can be placed in context where the effects of uncertainties arising in the definition of scenarios $(\mathrm{J})$, emissions projections $(\mathrm{B})$, background particulates $(\mathrm{N})$, or imported contributions (I) may far exceed the sensitivity of the model to FRAME matrices, which would render these uncertainties within FRAME relatively insignificant from the perspective of different policy scenarios. This is not to suggest that quantification of uncertainty in FRAME may be unnecessary as these are always useful for providing a more complete understanding of the dynamics of the system as a whole.

This conceptual framework is intentionally flexible to the extreme, facilitating the mapping of areas of uncertainty, together with linkages between them, in a manner which reflects the perspective and priorities of the observer. This will provide the observer with an understanding of uncertainties from their perspective which can be compared with equivalent maps from alternative perspectives, thus further enhancing understanding of the potential impacts of given policy scenarios. It highlights the linkages between components and the 'distance' (number of linkages) between different uncertainties and the observer. If, for example, a policy maker needs to understand the effect of road transport on aerosol concentrations, uncertainties in traffic projections can be quantified in a policy scenario. However, uncertainties in emissions factors (S - Figure 2) cannot; these may influence the model outputs, but are combined with and through many other components: with projections (V) to calculate emissions from road transport used by UKIAM - with further uncertainties in atmospheric dispersion (D) and other projections (B) - to calculate aerosol concentrations (M) which are re-combined with traffic emissions to derive roadside (W) and background air quality (X). The policy maker can, however, quantify the sensitivity of the results to alternative definitions of emissions factors, ceteris paribus.

In summary therefore, this conceptual framework enables both developers and users of integrated assessment models to map and understand relationships between different areas of uncertainty based upon:

- the source and type of uncertainty and its relationship to the overall system;

- its reflection of historical memory or projected futures;

- its utility in decision support or communication;

- the objective in relation to maximising precision, generality or reality [Levins, 1966]; and

- the associated 'mode of thought', ranging from the analytical through the experimental to the intuitive [Hammond, 1978].

Finally, this conceptual framework is not intended to 'compete' with other approaches to addressing uncertainty (eg HAZOP), but does provide an alternative (visual) and complementary approach which can be used alongside, for example, approaches which capture knowledge related uncertainties [Petersen, 2009].

\section{CONCLUSIONS}

This conceptual framework enables the 'observer' (policy maker, scientist etc.) to map different components of an integrated assessment model from a perspective which reflects their requirements for understanding uncertainty. It highlights the linkages between components and the 'distance' between different uncertainties and the observer, distinguishing whether an uncertainty is directly quantifiable from the observers' perspective, or whether it is the sensitivity of the system to a 'distant' uncertainty that provides the best understanding.

The generic nature of this framework means that the methodology is transferable. For example, the UKIAM may treat emissions projections as extraneous input. However, behind these projections will be a variety of models, data, expert opinion etc. which can equally be mapped onto this conceptual framework (with a focus on emissions as opposed to effects). Likewise, traffic projections will have emerged from complex transport demand models, socio-economic data etc. Very quickly an hierarchy of 'uncertainty maps' emerges from which the original 'problem' of how to deal with uncertainty can be understood in all its complexity.

Policy makers must still make the decisions. This generic conceptual framework will help scientists and modellers to provide policy makers with a better understanding of the uncertainties whilst highlighting that models are only heuristic tools designed to help make the decision and understand the potential impacts of that decision in an inherently uncertain world.

\section{REFERENCES}

Alcamo, J. \& Bartnicki, J., 1987, A framework for error analysis of a long-range transport model with emphasis on parameter uncertainty, Atmospheric Environment, 21(10), 2121-2131

Amann, M., Bertok, I., Borken-Kleefeld, J., Cofala, J., Heyes, C., Hoglund-Isaksson, L., Klimont, Z., Nguyen, B., Posch, M., Rafaj, P., Sandler, R., Schoepp, W., Wagner, F. \& Winiwarter, W., 2011, Cost-effective control of air quality and greenhouse gases in Europe: modelling and policy applications, Environmental Modelling \& Software, (in press)

ApSimon, H., Warren, R. \& Mediavilla-Sahagun, A., 2002, Applying Risk Assessment Techniques to Air Pollution Modelling \& Abatement Strategies, pp189-199, in [Borrego \& Sayers (eds), Air Pollution Modeling and Its Application XV, Kluwer Academic, New York, 2002, ISBN 978-0-306-47813-0]

Bush, T., Tsagatakis, I., Passant, N., Griffin, A. \& B. Pearson, 2010, UK Emission Mapping Methodology 2007, National Atmospheric Emissions Inventory, September 2010, AEAT/ENV/R/2863 
Carnevale, C., Finzi, G., Pisoni, E. \& Volta, M., 2009, Neuro-fuzzy and neural network systems for air quality control, Atmospheric Environment, 43, 4811-4821

Cullen, A.C. \& Frey, H.C., 1999. Probabilistic Techniques in Exposure Assessment: A Handbook for Dealing with Variability and Uncertainty in Models and Inputs. Plenum Press, New York, USA.

de Haan, P. \& Keller, M., 2004, Modelling fuel consumption and pollutant emissions based on real-world driving patterns: the HBEFA approach, Int. J. Environment \& Pollution, 22(3), 240-258

Derwent, R. G., 1987, Treating uncertainty in models of the atmospheric chemistry of nitrogen compounds, Atmospheric Environment, 21(6), 1445-1454

EC4MACS, 2010, Workshop on uncertainty treatment, IIASA, Laxenburg, 3-4 November 2010, www.ec4macs.eu

Entec, 2009, Multi-Pollutant Measures Database, Report to Defra, August 2009, Entec UK Limited

Fournier, N., Dore, A. J., Vieno, M., Weston, K. J., Dragosits, U. \& Sutton, M. A., 2004, Modelling the deposition of atmospheric oxidised nitrogen and sulphur to the United Kingdom using a multi-layer long-range transport model. Atmospheric Environment, 38(5), 683-694.

Hammond, K. R., 1978, Toward increasing competence of thought in public policy formation. In [Judgement and Decision in Public Policy Formation, ed. K.R. Hammond. AAAS Selected Symposium, Westview Press, Colorado, 11-32]

Jong, G., Daly, A., Pieters, M., Miller, S., Plasmeijer, R. \& Hofman, F., 2007, Uncertainty in traffic forecasts: literature review and new results for The Netherlands, J. Transportation, 34(4), 375-395

Kioutsioukis, I., Tarantola, S., Saltelli, A. \& Gatelli, D., 2004, Uncertainty and global sensitivity analysis of road transport emission estimates, Atmospheric Environment, 38, 6609-6620

Kouridis, C., Gkatzoflias, D., Kioutsioukis, I., Ntziachristos, L., Pastorello, C. \& Dilara, P., 2010, Uncertainty estimates and guidance for road transport emissions calculations, JRC Scientific \& Technical Report, Office for Official Publications of the European Communities, ISBN 978-92-79-15307-5

Levins, R., 1966, The strategy of model building in population biology. American Scientist 54 (4), 421-431.

Mathijssen, J., Petersen, A., Besseling, P., Rahman, A. \& Don, H. (eds.), 2007, Dealing with Uncertainty in Policy Making, CPB/PBL/Rand Europe, Final report of the conference Dealing with Uncertainty in Policy Making, 16-17 May 2006, The Hague, ISBN 978-90-6960-210-3

Oxley, T. \& ApSimon, H.M., 2007, Space, time and nesting Integrated Assessment Models, Environmental Modelling \& Software, 22, 1732-1749

Oxley, T., ApSimon, H., Dore, A., Sutton, M., Hall, J., Heywood, E., Gonzales del Campo, T., \& Warren, R., 2003a, The UK Integrated Assessment Model, UKIAM: A national scale approach to the analysis of strategies for abatement of atmospheric pollutants under the CLRTAP, Integrated Assessment, 4(4), 236-249

Oxley, T., ApSimon, H. \& Valiantis, M., 2011, Modelling national air quality strategy scenarios with UKIAM: uncertainties emerging from the integration of multiple spatial scales, Int. J. Environment \& Pollution, 44, 54-61

Oxley, T., Lemon, M., Jeffrey, P., 2003b. Indicators of socio-natural change: scientific meaning and contextual interpretation. Journal of Environmental Assessment Policy and Management 5(1), 1-26

Oxley, T., Valiantis, M., Elshkaki, A. \& ApSimon, H., 2009, Background, Road and Urban Transport modelling of Air quality Limit values (the BRUTAL model). Environmental Modelling and Software, 24, 1036-1050

Page, T., Whyatt, J.D., Beven, K.J. \& S.E. Metcalfe, 2004, Uncertainty in modelled estimates of acid deposition across Wales: A GLUE approach. Atmospheric Environment, 38, 2079-2090.

Passant, N. R., 2003, Estimation of uncertainties in the NAEI, Report to Defra, AEAT/ENV/R/1039/Issue1

Petersen, A. C., 2006, A philosophical study of computer-simulation uncertainties and their role in climate science and policy advice, PhD Thesis, Antwerpen, ISBN 9789055892808

Reinds, G. \& de Vries, W., 2010, Uncertainties in critical loads and target loads of sulphur and nitrogen for European forests: analysis and quantification, Science of the Total Environment, 408(8), 1960-1970

Schoepp, W., Klimont, Z., Suutari, R. \& Cofala, J., 2005, Uncertainty analysis of emissions estimates in the RAINS integrated assessment model, Environmental Science \& Policy, 8, 601-613

Skeffington, R. A., 2006, Quantifying uncertainty in critical loads: literature review, Water, Air \& Soil Pollution, 169, 3-24

Stocker, J. \& Carrusters, D., 2007, Road vehicle non-exhaust particulate matter: Initial air quality model development and application, model uncertainty analysis and further model improvements, TRL \& CERC, CERC Report FM668/R01/06

Sutton, M., Simpson, D., Levy, P., Smith, R., Reis, S., van Oijen, M. \& de Vries, W., 2008, Uncertainties in the relationship between atmospheric nitrogen deposition and forest carbon sequestration, Global Change Biology, 14, 2057-2063

Tost, H., Lawrence, M. G., Bruhl, C., Jockel, P., et al., 2010, Uncertainties in atmospheric chemistry modelling due to convection parameterisations and subsequent scavenging, Atmos. Chem. Phys., 10, 1931-1951

Vieno, M., Dore, A.J., Bealey, W.J., Stevenson D.S. \& M.A. Sutton, 2010, The importance of source configuration in quantifying footprints of regional atmospheric sulphur deposition. Science of the Total Environment, 408(4), 985-995

Wolniewicz, M. B. \& Aherne, J., 2010, Development of an approach to assess uncertainty in terrestrial critical load and exceedance, Report to Canadian Council of Ministers on the Environment, CCME Contract 479-2010

Walker, W. E., Harremoës, P., Rotmans, J., Van der Sluijs, J. P., van Asselt, M. B. A., Janssen, P. H. M. \& Krayer von Krauss, M. P., 2003, Defining Uncertainty: A conceptual basis for uncertainty management in model-based decision support, Integrated Assessment, 4(1), 5-17

Warren, R. \& ApSimon, H., 1999, Uncertainties in integrated assessment modelling of abatement strategies: illustrations with the ASAM model, Environmental Science \& Policy, 2, 439-456

Zak, S., Beven, K. \& Reynolds, B., 1997, Uncertainty in the estimation of critical loads: A practical methodology, Water Air \& Soil Pollution, 98(3-4), 297-316

Zhao, Y. \& Kockelman, K. M., 2002, The propagation of uncertainty through travel demand models: an exploratory analysis, Annals of Regional Science, 36(1), 145-163 